Military Technical College Kobry El-Kobbah, Cairo, Egypt

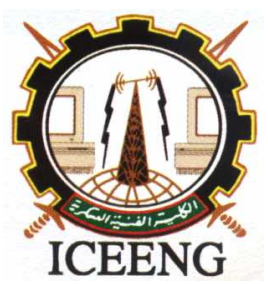

\author{
$6^{\text {th }}$ International Conference \\ on Electrical Engineering \\ ICEENG 2008
}

\title{
Two-Phase Approach Based on Genetic Algorithm for Reactive Power Dispatch Problem
}

\author{
By
}

A. A. Mousa*

\section{Abstract:}

The problem of reactive power dispatch (RPD) is to allocate reactive power generation so as to minimize the real power transmission losses and keep all voltage within the limits, while satisfying a number of equality and inequality constraints. This paper presents a new methodology for solving RPD. This methodology is consists of two phases. The first one employs the genetic algorithm (GA) to obtain a feasible solution subject to desired load convergence, while the other phase employs efficient GA to obtain the optimal solution. Also, some major improvements are added to the traditional genetic algorithm in order to improve the convergence and to find a better solution. Extensive testing of the proposed algorithm is done on standard IEEE-30 bus system and the results have been compared to those reported in the literature. The comparison demonstrates the superiority of the proposed approach and confirms its potential to solve the RPD problem.

\section{Keywords:}

Reactive Power Dispatch Problem; Genetic Algorithm; Nonlinear Programming.

* Department of basic engineering science, Faculty of Engineering, Shebin El-Kom, Menoufia University, Egypt. E-Mail: A mousa15@yahoo.com 


\section{Introduction}

Voltage stability condition is a crucial aspect in the power system operation and planning. The stressed condition in a power system caused by reactive power loading has made the system operating close to its stability limit while reducing the voltage on a particular load bus. Hence some measures should be taken in order to improve the voltage stability condition in the electric power system. Reactive Power Planning includes the reactive power dispatch, capacitor placement on the load bus to improve local voltage profile in the radial system.

A number of techniques ranging from classical techniques like gradient-based optimization algorithms to various mathematical programming techniques have been applied to solve this problem [4-10,15,16,19]. In most of these approaches the problem is linearised and then linear programming is used to solve the resulting optimization problem. This approximation is necessitated by the fact that these techniques have severe limitations in handling non-linear, discontinuous functions and constraints, and functions having multiple local minima, as is the case with RPD. The enhanced modeling and search power of the evolutionary algorithms (EA) developed recently has encouraged their application to the RPD problem [11-14,22,23]. EA include evolutionary programming (EP), genetic algorithms (GA) and evolutionary strategies (ES) [18].

An application of GA for the RPD problem is reported in [11]. The method decomposes the system into a number of sub-systems and employs interbreeding between the sub-systems to generate new solutions. All the controller states, including those with a continuous nature, are discretized and represented as integer values. Another approach based on a modified simple genetic algorithm is reported in [13]. The population selection and reproduction uses Benders' cut in the decomposed system and successive linear programming is used to solve the operational optimization subproblems. An EP approach for solving RPD is presented in [14]. The technique uses a floating point representation for control variables, thus avoiding the approximation introduced in binary representation of controllers in GA based approaches. An inner loop is used for function minimization without any consideration for constraints. Constraint satisfaction is carried out in an outer loop. Non-feasible solutions in the outer loop are rejected by attaching a penalty to their fitness values. A hybrid approach for solving RPD is presented in [3]. The method is based on evolutionary strategy (ES) i.e. mutation is the dominant search operator supported by crossover and a local improvement heuristic.

This paper proposes a new methodology for solving RPD. This methodology is divided into two phases. The first one employs the genetic algorithm (GA) to obtain a feasible solution subject to desired load convergence, while the other part employs GA 
to obtain the optimal solution. The standard IEEE 30-bus 6-genrator test system then used to verify the validity of the proposed approach.

This paper is organized as follows; problem formulation is reviewed in section 2 . Section 3 gives out the mechanism of Genetic algorithms(GAs). The proposed approach is presented in section 4. Implementation of the proposed approach are discussed in section 5. Conclusion follows in section 6.

\section{Problem Formulation}

The problem of reactive power dispatch (RPD)[2,15] is to allocate reactive power generation so as to minimize the real power transmission losses and keep all the voltages within the limits, while satisfying a number of equality and inequality constraints including the power flow equations, upper and lower voltage limits and capacity restrictions in various reactive power sources, generators and shunt capacitor banks. Mathematically, the problem can be stated as

$$
\text { Min } \mathrm{P}_{\mathrm{L}}=\sum_{i=1}^{N \text { bus }} P_{g i}-\sum_{i=1}^{N \text { bus }} P_{D i}
$$

S.t.

$$
\begin{gathered}
P_{g i}-P_{D i}-v_{i} \sum_{j=1}^{N b u s} V_{j}\left(G_{i j} \cos \delta_{i j}+B_{i j} \sin \delta_{i j}\right)=0, \mathrm{i}=1, \ldots, \text { Nbus } \\
Q_{g i}-Q_{D i}-v_{i} \sum_{j=1}^{N b u s} V_{j}\left(G_{i j} \sin \delta_{i j}+B_{i j} \cos \delta_{i j}\right)=0, \mathrm{i}=1, \ldots, \text { Nbus } \\
Q_{g i \min } \leq Q_{g i} \leq Q_{g i \max }, \quad \mathrm{i}=1: \mathrm{Ng} \\
Q C_{g i \min } \leq Q C_{g i} \leq Q C_{g i \max }, \quad \mathrm{i}=1: \mathrm{Ncap} \\
V_{i \text { min }} \leq V_{i} \leq V_{i \text { max }}, \quad \mathrm{i}=1: \mathrm{Nbus}
\end{gathered}
$$

where 


$\begin{array}{ll}\text { Nbus } & \text { Number of busbars } \\ \mathrm{NI} & \text { Number of transmission lines } \\ \mathrm{Ng} & \text { Number of generator } \\ \mathrm{Nt} & \text { Number of transformers } \\ \mathrm{Ncap} & \text { Number of shunt capacitors } \\ V_{i} & \text { Voltage magnitude at } i \text { th bus } \\ \delta_{i} & \text { Voltage angle at } i \text { th bus } \\ \delta_{i j} & \delta_{i}-\delta_{j} \\ r_{j k} & \text { Series resistance connecting buses } j \text { and } k \\ P_{g i}, Q_{g i} & \text { Real and reactive power generation at } i t h \text { bus } \\ P_{D i}, Q_{D i} & \text { Real and reactive power load at } i t h \text { bus } \\ Y_{i j} \angle \theta_{i j}=G_{i j}+j B_{i j} & i j t h \text { element of bus admittance matrix } \\ Q_{g i \min }, Q_{g i \max } & \text { Reactive power limits of } i t h \text { generator } \\ Q C_{i} & \text { Reactive power generated by } i t h \text { shunt capacitor bank } \\ Q C_{i \min }, Q C_{i \max } & \text { Reactive power limits of } i t h \text { shunt capacitor bank }\end{array}$

Thus, RPD is a complex combinatorial optimization problem involving non-linear functions having multiple local minima and non-linear and discontinuous constraints.

\section{Genetic Algorithm (GA)}

GA, invented by Holland [17] in the early 1970s, as a stochastic global search method that mimics the metaphor of natural biological evaluation. GAs operates on a population of candidate solutions encoded to finite bit string called chromosome. In order to obtain optimality, each chromosome exchanges information by using operators borrowed from natural genetic to produce the better solution. Figure1 shows Outline of GAs for optimization problems. The GAs differ from other optimization and search procedures in four ways $[1,20]$ :

(1) GAs work with a coding of the parameter set, not the parameters themselves. Therefore GAs can easily handle the integer or discrete variables.

(2) GAs search from a population of points, not a single point. Therefore GAs can provide a globally optimal solutions.

(3) GAs use only objective function information, not derivatives or other auxiliary knowledge. Therefore GAs can deal with the non-smooth, non-continuous and nondifferentiable functions which are actually existed in a practical optimization problem. 
(4) GAs use probabilistic transition rules, not deterministic rules

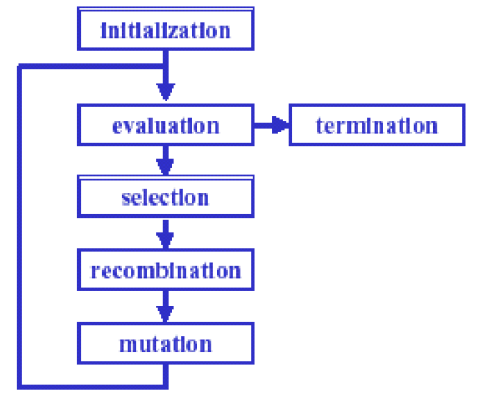

Fig.1:Outline of GAs for optimization problems

\section{The Proposed Approach}

In this section we present a novel optimization algorithm to solve the RPD problem formulated in the previous section. The solution is based on concept of co-evolution and repair algorithm for handling nonlinear constraints. The algorithm is consists of two phases. The first phase are: finding an initial feasible point by minimizing a function that measures the maximum violation of the constraints (Load flow equations), while the second phase employs efficient co-evolutionary algorithm for solving the resulting nonlinear programming problem (NLP), which combines concept of co-evolution, repairing procedure and elitist strategy.

\subsection{Solution Representation}

The algorithm uses a floating point representation for potential solutions. Each generation contain both feasible and infeasible individuals and we distinguish between them using flag pointer assigned to each individual

\subsection{Initialization Stage}

The population vectors in the first generation are initialized randomly satisfying the search space $\mathrm{S}$ (the lower and upper bounds), while elitist individual is initialized by zero. The algorithm needs initial system precisione, which enable the algorithm to initially locating an initial feasible point (reference point) that satisfying all constraint with the initial system precision. Also, for every generation the algorithm searches for updated reference point, updated reference point represents the individual with the minimum violation.

\subsection{Repairing Infeasible Individuals:}


The idea of this technique is to separate any feasible individuals in a population from those that are infeasible by repairing infeasible individuals. This approach co-evolves the population of infeasible individuals until they become feasible. New feasible individuals $(\mathrm{z})$ are generated on a segment defined by two points feasible individual (i.e., initial reference point $\left.\xi^{t} \in \mathrm{F}\right)$ and infeasible individuals $\left(\omega^{t}\right)$, But the segment may be extended equally on both sides determined by a user specified parameter $\mu[19,20]$. Thus, a new feasible individual is expressed as:

$$
z_{1}=\gamma \cdot \omega^{t}+(1-\gamma) \cdot \zeta^{t}, \quad z_{2}=(1-\gamma) \cdot \omega^{t}+\gamma \cdot \zeta^{t}
$$

Where $\gamma=(1+2 \mu) \delta-\mu$ and $\delta \in[0,1]$ is a random generated number. Figure 2 gives schematic view of possible sampling region for the generated individuals.

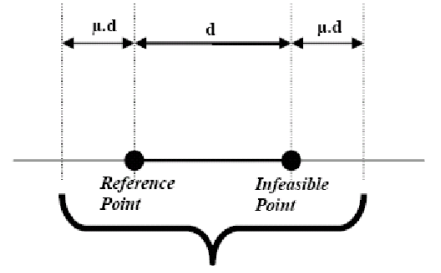

Fig. 2. Possible sampling region

\subsection{Elitist strategy.}

Using an elitist strategy to produce a faster convergence of the algorithm to the optimal solution of the problem. The elitist individual represents the more fit individual of the population. The use of elitist individual guarantees that the best fitness individual never increase (Minimization problem) from one generation to the next .

\subsection{Evolution process stage:}

To reduce the violations of the constraints in phase-I to an acceptable level (desired precision $\varepsilon^{*}$ ), further optimization is necessary. This involves minimizing a function that measures the maximum violation of the constraints. These minimizations can be done using $l_{\infty}$ norm as objective function to evaluate fitness for each individual, where the distance from the system precision $\varepsilon$ to desired precision $\varepsilon^{*}$ should be minimized.

$$
\min \left\|\varepsilon_{\mathrm{i}}-\varepsilon_{i}{ }^{*}\right\|_{\infty}=\min \left(\max _{\mathrm{i}}\left|\varepsilon_{\mathrm{i}}-\varepsilon_{i}{ }^{*}\right|\right)
$$

The algorithm applies tournament selection procedure/roulette wheel selection to construct the new population. 


\subsection{Stopping Rule:}

The algorithm is terminated for either one of the following conditions is satisfied:

- The maximum number of generations is achieved.

- When the genotypes (the genotypes structures) of the population of individuals converges, convergence of the genotype structure occur when all bit positions in all strings are identical, in this case, crossover will have no further effect.

\subsection{Proposed Approach For RPD}

In solving the RPD, two phases of the algorithm need to be identified, phase-I implements GA to find an initial feasible point, while phase-II employs efficient coevolutionary algorithm for solving the resulting NLP. Figure 3 describes the main steps of the proposed algorithm.

\section{Algorithm Procedure}

Begin

Input $\varepsilon \in R^{2^{*} \text { Nbus }}$ (initial system precision), $\varepsilon^{*} \in R^{2^{*} \text { Nbus }}$ (desired precision)

Population initialization:

Get a feasible point( initial reference point ) $\xi^{t=0}$;

\section{PHASE I}

While $\left(\varepsilon>\varepsilon^{*}\right)$ do

Begin

\section{End}

$$
\begin{aligned}
& \text { Select } P^{t} \text { from } P^{t-1} \text {; } \\
& \text { Keep the best; } \\
& \text { Perform recombination } P^{t} \text {; } \\
& \text { Repair population; } \\
& \text { Check (Stopping criteria); } \\
& \text { Elitist; }
\end{aligned}
$$

Get a feasible point( initial reference point ) $\xi^{t}$;

\section{PHASE II}

$\mathrm{T}=0$;

Population initialization:

Begin

repair population;

Keep the best; 


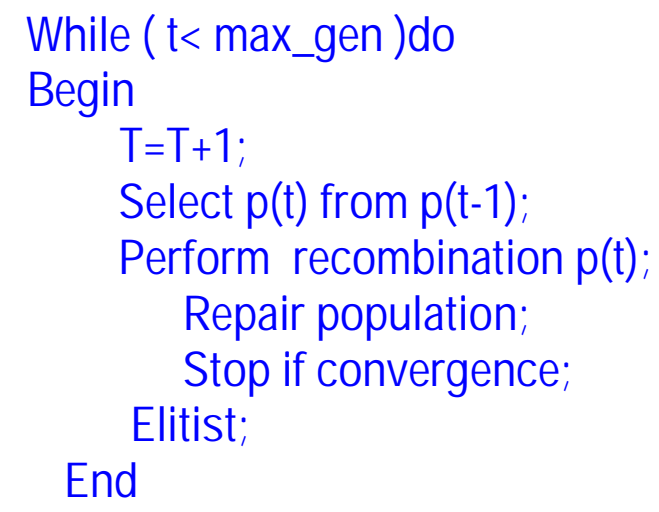

End

End

Fig. 3. The structure of optimization system

\section{Implementation Of The Proposed Approach}

\section{System Data}

The described methodology is applied to the standard IEEE 30-bus 6-generator test system to investigate the effectiveness of the proposed approach. The single-line diagram of this system is shown in Figure 4 and the detailed data for this system are given in [24]. The techniques used in this study were developed and implemented on 2.7-MHz PC using MATLAB environment. Table1 lists the parameter setting used in this study.

\begin{tabular}{ll} 
Table 1 : GA parameters & \\
\hline Population size (N) & 150 \\
No. of Generation & 120 \\
Crossover probability & 0.95 \\
Mutation probability & 0.03 \\
Selection operator & Tournament / Roulette \\
& Wheel \\
Crossover operator & BLX- $\alpha$ \\
Mutation operator & Polynomial mutation \\
\hline
\end{tabular}




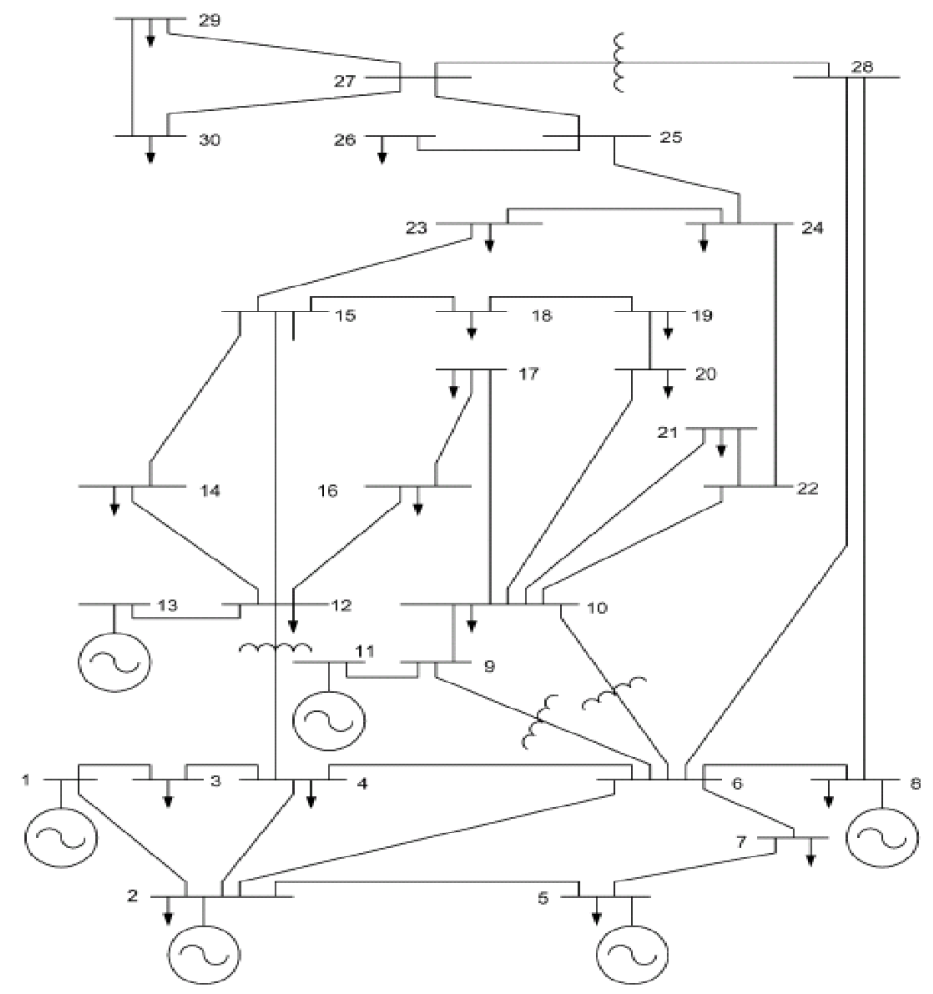

Fig. 4. Single line diagram of IEEE 30-bus 6-generator test system

\section{Results And Discussions}

After running the load flow analysis from a flat voltage start, the generated power and network power loss are obtained as follows

$$
\begin{aligned}
& \sum P_{G}=2.893857 \text { p.u. } \\
& \sum Q_{G}=0.980199 \text { p.u. } \\
& P_{\text {Loss }}=0.059879 \text { p.u. }
\end{aligned}
$$

Figure 5 shows the convergence curve for 120 generation of the proposed approach

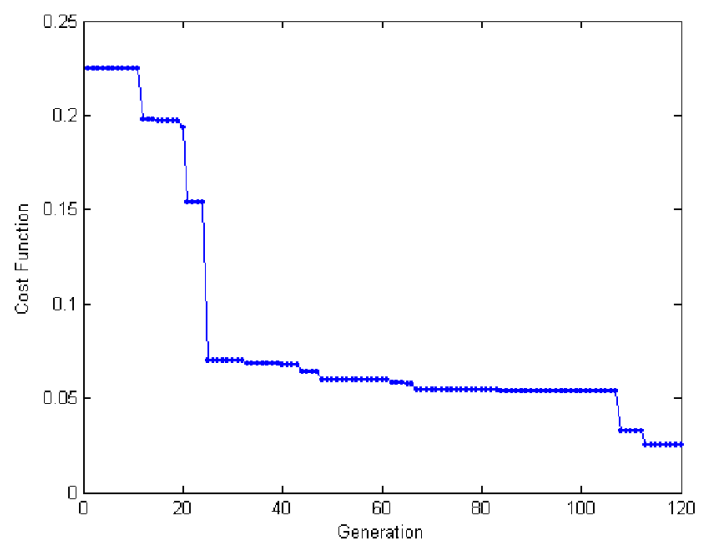

Fig.5. convergence of cost with 120 generations 
Figure 6-7 summarizes the results of the optimal controller settings (bus voltage and reactive power sources) as obtained by the proposed approach.

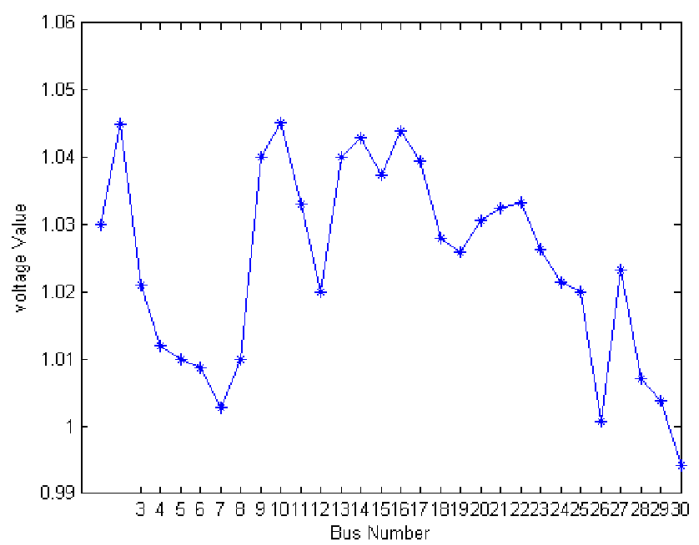

Fig. 6. Optimal setting of bus voltage obtained by the proposed method.

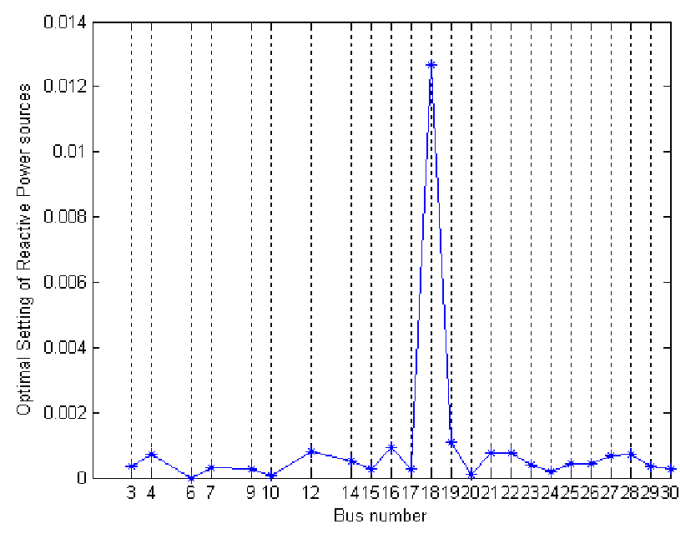

Fig. 7. Optimal setting reactive power resources obtained by the proposed method.

Table 2 summarizes the results of the optimal controller settings as obtained by different methods given in [3] and limit violations in load bus voltages (Vviolation) and generator reactive power outputs (Qviolation) caused by these methods. These results show that maximum saving is obtained by the proposed approach. At the same time, this method succeeds in keeping the dependent variables within their limits.

As hardware and the software environments affect the computational time significantly, it is not possible to compare the computational time requirements of different methods unless all the methods are programmed using the same environment and run on the same hardware. However, repeated load flow executions are the main time consuming computations in all these

methods. Therefore, the total number iterations (generation) is a reasonable basis for comparing the computational performance. The proposed method requires considerably less number of iterations and is, therefore, faster than the other methods.

Table 2. Comparison of optimal transmission loss for different methods

\begin{tabular}{|l|c|c|c|c|c|c|}
\hline Method & $\sum P_{G}$ & $P_{L}$ & $P_{\text {Swer }}$ & $\% P_{\text {Swer }}$ & $V_{\text {violation }}$ & $Q_{\text {violation }}$ \\
\hline CGA[3] & 2.88380 & 0.04980 & 0.01008 & 16.84 & Nil & Nil \\
\hline AGA[3] & 2.88326 & 0.04926 & 0.01062 & 17.74 & Nil & Nil \\
\hline EP1[3] & 2.88362 & 0.04963 & 0.01025 & 17.12 & Present & Nil \\
\hline
\end{tabular}




\begin{tabular}{|l|c|c|c|c|c|c|}
\hline EP2[3] & 2.88414 & 0.05015 & 0.00972 & 16.23 & Present & Nil \\
\hline Broyden [3] & 2.89135 & 0.05736 & 0.00252 & 04.21 & Nil & Nil \\
\hline HES [3] & 2.88270 & 0.04870 & 0.01115 & 18.63 & Nil & Nil \\
\hline $\begin{array}{l}\text { Proposed } \\
\text { Approach }\end{array}$ & 2.85910 & 0.02510 & 0.03478 & 58.08 & Nil & Nil \\
\hline
\end{tabular}

\section{Conclusions}

The problem of reactive power dispatch is to allocate reactive power generation so as to minimize the real power transmission losses and keep all voltage within the limits, while satisfying a number of equality and inequality constraints. This paper presents a new methodology for solving RPD. This methodology is consists of two-phases. The first one employs the genetic algorithm (GA) to obtain a feasible solution subject to desired load convergence, while the other phase employs efficient GA to obtain the optimal solution. The standard IEEE 30-bus 6-genrator test system then used to verify the validity of the proposed approach. The result confirms the proposed approach potential to solve the RPD problem. The main features of the proposed algorithm could be summarized as follows:

a) The proposed technique has been effectively applied to solve the RPD.

b) Allowing a decision maker to control the precision of load flow equations by defining desired system precision $\varepsilon^{*} \in \boldsymbol{R}^{2^{*} \mathrm{Nbus}}$ values.

c) The proposed approach is suitable to complex problems, where the feasible region $F$ is very small with respect to the search space s (i.e., $\frac{F}{S} \ll$ ).

d) Low computational time where, the computational time grows with the number of iterations

e) Empirical results show that our approach is very efficient against other recent approaches for solving RPD.

\section{References}

[1] M. A. Abido, Multiobjective evolutionary algorithms for electric power dispatch problem, IEEE Trans. On Evolutionary Computation, Vol. 10, No.3, June 2006.

[2] M. Azzam and A.A.Mousa, Using genetic algorithm and topsis technique for Multiobjective reactive power compensation, Journal of Engineering Sciences, Assiut University, Vol. 35, No. 3 pp. 783-797, May 2007.

[3] D. Bhagwan Das and C. Patvardhan, A new hybrid evolutionary strategy for reactive power dispatch, Electric Power Systems Research 65 (2003) 83-90. 
[4] J.M. Bright, K.D. Demaree, J.P Britton, Reactive security and optimality in real time, IFAC Power Systems and Power Plant Control, Beijing (1986) 65-70.

[5] B. Cova, N. Losignore, P. Marannino, M. Montagna, Contingency constrained optimal reactive power flow procedures for voltage control in planning and operation, IEEE Transactions on Power Systems 10 (2) (1989) 602-608.

[6] N. Deeb, S.M. Shaidepour, Linear reactive power optimization in a large power network using the decomposition approach, IEEE Transactions on Power Systems 5 (2) (1990) 428-435.

[7] J. Goossens, Reactive power and system operation-incipient risk of generator constraints and voltage collapse, Invited Paper, IFAC Power Systems and Power Plant Control, Seoul, Korea, 1989, 1-10.

[8] S. Granville, Optimal reactive dispatch through interior point methods, IEEE Transactions on Power Systems 9 (1) (1994) 136-146.

[9] J.S. Horton, L.L. Grigsby, Voltage optimization using combined linear programming and gradient techniques, IEEE Transactions on PAS 103 (7) (1984) 1637-1643.

[10] M. Huneault, F.D. Galiana, A survey of the optimal power flow literature, IEEE Transactions on Power Systems 6 (2) (1991) 762-770.

[11] K. Iba, Reactive power optimization by genetic algorithm, IEEE Transactions on Power Systems 9 (2) (1994) 685-692.

[12] L.L. Lai, J.T. Ma, Application of evolutionary programming to reactive power planning-comparison with nonlinear programming approach, IEEE Transactions on Power Systems 12 (1) (1997) 198-206.

[13] K.Y. Lee, Y.M. Park, Optimization method for reactive power planning by using a modified simple genetic algorithm, IEEE Transactions on Power Systems 10 (4) (1995) 1843-1850.

[14] J.T. Ma, L.L. Lai, Evolutionary programming approach to reactive power planning, IEE Proceeding-Generation Transmission Distribution 143 (4) (1996) 365-370.

[15] K.R.C. Mamandur, R.D. Chenoweth, Optimal control of reactive power flow for improvements in voltage profiles and for real power loss minimization, IEEE Transactions on Power Systems PAS 100 (7) (1981) 3185-3193.

[16] J.R.S. Manlovani, A.V. Garcia, A heuristic method for reactive power planning, IEEE Transactions on Power Systems 11 (1) (1995) 68-74.

[17] Z. Michalewicz, Genetic Algorithms + Data Structures = Evolution Programs, Springer-Verlag, 3rd Edition, (1996).

[18] V. Miranda, D. Srinivasan, L.M. Proneca, Evolutionary computation in power systems, Electrical Power and Energy Systems 20 (2) (1998) 89-98.

[19] M.S. Osman, M.A. Abo-Sinna, and A.A. Mousa, IT-CEMOP: An Iterative Coevolutionary Algorithm for Multiobjective Optimization Problem with Nonlinear Constraints, Journal of Applied Mathematics \& Computation (AMC) 183(2006)373389. 
[20] M.S. Osman, M.A. Abo-Sinna, and A.A. Mousa, A Solution to the Optimal Power Flow Using Genetic Algorithm, Applied Mathematics\&Computation 2004; 155(2004)391-405.

[21] L.D.B. Terra, M.J. Short, A global approach for VAR/voltage management, IFAC Power Systems and Power Plant Control, Seoul, Korea (1989) 81-86.

[22] Q.H. Wu, Y.J. Cao, J.Y. Wen, Optimal reactive power dispatch using an adaptive genetic algorithm, Electrical Power and Energy Systems 20 (8) (1998) 563-569.

[23] Q.H. Wu, J.T. Ma, Power system optimal reactive power dispatch using evolutionary programming, IEEE Transactions on Power Systems 10 (3) (1995) 1243-1249.

[24] R. Zimmerman, Gan D., MATPOWER: A Matlab power system simulation package, Available: http://www.pserc.cornell.edu/matpower/ 